\title{
Combined hardening parameters of steel CK45 under cyclic strain-controlled loading: Calibration methodology and numerical validation
}

\author{
B. Paygozar ${ }^{1 *}$, S.A. Dizaji1 ${ }^{1}$ M.A. Saeimi Sadigh² \\ 1 Department of mechanical engineering, TED University, Ankara, Turkey \\ Phone: +90-312-585-0270 \\ 2 Department of Mechanical Engineering, Azarbaijan Shahid Madani University, Tabriz, Iran
}

\begin{abstract}
This study is to indicate the methodology of investigating the behavior of materials in the plastic domain while bearing cyclic loading i.e. low cycle fatigue. Materials under such loading, which experience huge amount of plastic deformation, are affected by the hardening or softening effects of loading which should be taken into account in all applications and numerical simulations as well. This work investigates the methodology of obtaining the nonlinear isotropic and kinematic hardening of steel CK45. To find the parameters of the above mentioned combined nonlinear isotropic/kinematic hardening one tensile test as well as three strain-controlled low cycle fatigue tests are carried out to extract the monotonic stress/strain curve and three diagrams of hysteresis curves, respectively. Then, four parameters necessary to simulate the nonlinear isotropic/ kinematic behavior of the material are extracted by means of curve fitting technique using MATLAB software. Afterwards, the accuracy of the data extracted from the experimental tests using the proposed methodology, are verified in a finite element package, ABAQUS, through implementing two user defined subroutines UMAT written in FORTRAN. It is indicated that the computed constants draw stress-strain curves much closer to experimental responses than isotropic hardening model does. Eventually, the numerical results acquired by simulating the behavior of the sample under cyclic loading with importing the constants, calculated via combined hardening model, to ABAQUS reflects results highly close to the experimentally obtained response of the sample. It means that the procedure used to find the constants is accurate enough and consequently the constants computed are able to be used in both ABAQUS and subroutines.
\end{abstract}

ARTICLE HISTORY

Revised: 22nd Dec 2019

Accepted: $8^{\text {th }}$ Jan 2020

\section{KEYWORDS}

Combined hardening;

Chaboche model;

stabilized hysteresis

curves.

\section{INTRODUCTION}

In all applications of metallic material under cyclic loading that force the material to experience the plastic deformations, the use of combined isotropic/kinematic hardening model is obligatory [1, 2]. This is because the Bauschinger effect shown in such loading should be taken into account. To model this behavior of materials, implementing only the isotropic hardening model is somewhat inaccurate while improving the model by adding the kinematic hardening can better the estimations of the materials behavior- in other words, applying nonlinear combined hardening models give an exact prediction of the material performance [3]. One popular one of such models is the Ramberg-Osgood model which considers this part of materials behavior [4]. This model copes with both elastic and plastic areas with the help of stress-strain curves. Ziegler [5] as well as Prager [6] hardening laws are two other suitable models in considering the plastic behavior of materials. According to the last introduced model, Armstrong and Frederic [7] introduced a nonlinear kinematic-based model. In modelling isotropic kind of hardening, Voce hardening law can have good outcomes due to including two different terms- a linear term as well as a nonlinear asymptotic term [8].

In the recent studies, effects of the hardening has been investigated through some well-known, practical models such as isotropic hardening and Chaboche combined hardening. For instance, Zhuang et al. [9] examined the hardening response of Al2024 aluminum alloy specimens when loaded under complex non-proportional conditions. They found out that the hardening behavior of the samples obtained by combined hardening model is of higher precision than that acquired through isotropic hardening model. Moreover, they proved that employing combined hardening model along with fatiguebased damage accumulation enhances the predictability of the fracture onset when the materials enduring torsion-tension loading. In a similar study, low cycle fatigue behavior of steel samples in addition to the response of low cycle creepfatigue were investigated by Zhao et al. [10]. They used isotropic hardening model as well as a modified combined kinematic hardening model and a proposed constitutive model for non-linear interaction damage accumulation so as to depict the hysteresis loops of stress-strain data up to fracture. This method was used to estimate the failure life with respect to changes done in the applied strain amplitudes and duration periods. They introduced their modified model appropriately practical in describing the accelerated cyclic softening of the materials and the reduction of their failure lives. 
In a micro-mechanical investigation carried out by Aghaei and Ziaei-Rad [11] on dual-phase steels, Lemaitre damage model in addition to combined isotropic/kinematic hardening were utilized simultaneously in order to simulate the behavior of the material numerically. Scanning electron microscopy used to do a plain strain analysis in a chosen representative volume element captured from the surface of the specimen. The analyses along with simulations indicate that most of damaged regions were located in ferrite/ martensite boundary. It was shown that damage locations are especially situated between two martensite islands, really close to each other. These regions can easily be found in main shear bands areas. Hence, such bands are the places, in which damage usually initiates and grows.

Modeling of anisotropic hardening considering Bauschinger effect experienced in sheet metals under cyclic loading was surveyed by Yoshida et al. [12]. They proposed a solution, as a constitutive model of plasticity, to represent the evolution of anisotropy and the Bauschinger effect in a cyclic loaded sheet metals. As a solution, an anisotropic yield function, successively changed with plastic strain augmentations, was defined as an interpolation between two yield functions at two discrete levels of plastic strain. They utilized the combined anisotropic-kinematic hardening model of large-strain cyclic plasticity with small elastic strain. It was found out that when a polynomial sort of yield function is used, the proposed model is simplified to the interpolation of its material parameters. Moreover, experiments gave some kinematic hardening parameters independent of anisotropic hardening parameters. Another similar work, by Lee et al. [13], employed combined anisotropic and distortion hardening laws to explain directional behavior using Bauschinger effect. In the research, directional anisotropic hardening of the material loaded under the non-proportional condition was modeled. They previously proposed a new coupled yield function $[14,15]$ to obtain directional hardening behavior, which was improved in this study, to demonstrate the directional hardening and Bauschinger effect as well.

In this paper, the plastic behavior of steel CK45 is declared with the help of combined hardening model. Chaboche nonlinear kinematic hardening model as well as Voce isotropic hardening model were utilized to extract the necessary parameters in order to predict the exact behavior of the material under strain-controlled low cyclic fatigue loading. These parameters are computed through curve fitting technique utilizing two equations fitted to some data extracted from a monotonic stress/strain curve and three stabilized hysteresis loops.

\section{METHODS AND MATERIALS}

\section{Nonlinear Hardening Models}

The model utilized in present study is a combination of isotropic and kinematic hardening models, which was introduced by Armstrong and Frederic [7] for the first time and subsequently improved by Chaboche [16, 17]. As mentioned previously, the combined model takes both isotropic and kinematic hardening into account, simultaneously. Figure 1 indicates the translation and expansion of the yield surface in accordance with plastic strain in more detailed form.

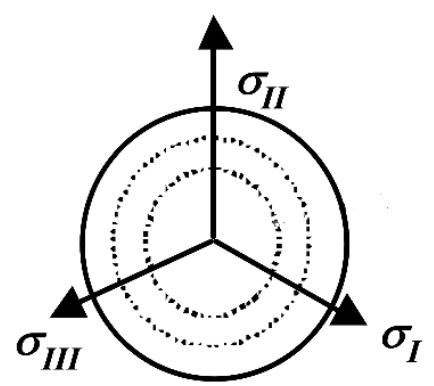

(a)

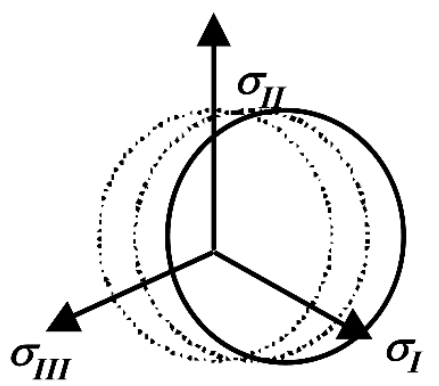

(b)

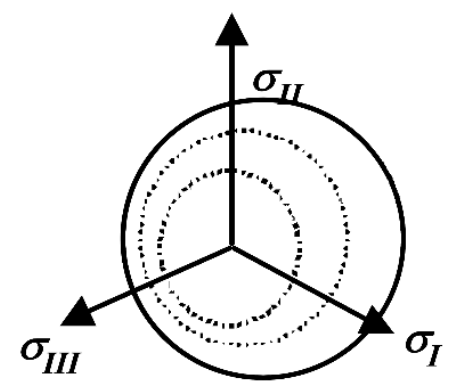

(c)

Figure 1. Yield surface adaptation in accord with plastic deformation in principal stress space [18]: (a) Isotropic hardening, (b) kinematic hardening and (c) combined hardening.

According to this model and considering von Mises yield criterion, the yield surface can be expressed as below:

$$
F=\sqrt{\frac{3}{2}\left(\sigma_{i j}^{\prime}-\alpha_{i j}^{\prime}\right):\left(\sigma_{i j}^{\prime}-\alpha_{i j}^{\prime}\right)}-\sigma_{s}^{2}\left(\varepsilon_{e q}^{p}\right)=0
$$

where $\sigma^{\prime}, \alpha^{\prime}$ and $\sigma_{s}$ are the deviatoric tensor, back stress tensor and current yield stress, respectively. The isotropic part of the model can be established by the exponential Voce's equation as below [8]:

$$
\sigma_{s}=\sigma_{s}^{0}+A\left(1-e^{-b \varepsilon_{e q}^{p}}\right)
$$


where $\sigma_{s}^{0}$ is the initial yield stress. In addition, the kinematic part can develop gradually through the equation which can be written as follow [19]:

$$
d \alpha_{i j}=\frac{C}{\sigma_{s}}\left(\sigma_{i j}-a_{i j}\right) d \varepsilon_{p}-\gamma \alpha_{i j} d \varepsilon_{p}
$$

where $\alpha_{i j}$ reflects the back stress and $\mathrm{C}$ and $\gamma$ are two constants related to the kinematic hardening of the material. Hence, the unknown material parameters of the study increased to four including $\mathrm{A}, \mathrm{b}, \mathrm{C}$ and $\gamma$. The procedure for obtaining or calibrating the parameters will be described later on.

\section{Experimental Procedure}

To obtain the monotonic stress-strain curve of steel CK45, one sample was manufactured and tested according to ASTM E8/E8M-09 and by using a universal tensile test machine (see Figure 2a). The result of this test was utilized to find two parameters of the nonlinear combined hardening model, which relate to its isotropic part. Figure 3 shows the monotonic diagram of engineering stress/strain. In addition, all mechanical properties of the material extracted from the diagram were gathered in Table 1.

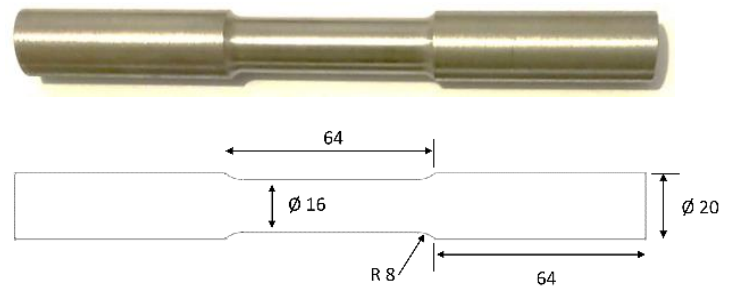

(a)

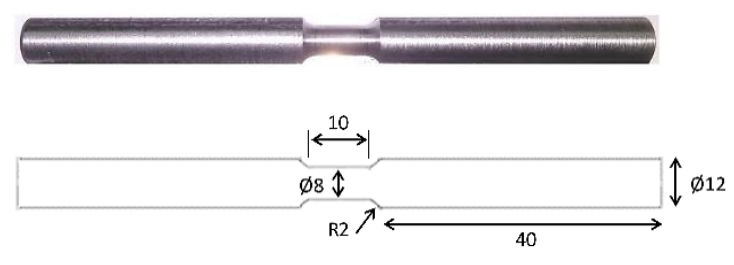

(b)

Figure 2. The specimens used to extract: (a) the monotonic stress-strain curve and (b) the hysteresis loops of the straincontrolled loading.

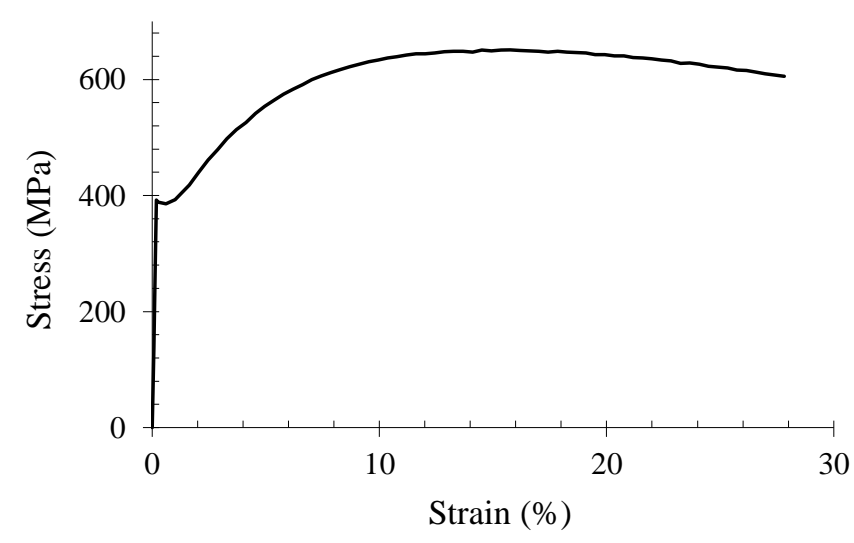

Figure 3. Monotonic stress-strain curve of steel CK45 obtained from tensile test.

Table 1. Mechanical properties of steel CK45.

\begin{tabular}{cc}
\hline Parameter & Quantity \\
\hline Young's modulus & $202[\mathrm{GPa}]$ \\
Yield stress & $393[\mathrm{MPa}]$ \\
Ultimate stress & $637[\mathrm{MPa}]$ \\
Elongation at failure & $27.8 \%$ \\
\hline
\end{tabular}

On the other hand, three samples were machined in order for obtaining stabilized hysteresis cycles under fatigue test. Figure $2 b$ shows the dimensions of the above-mentioned samples in detail [20]. For fatigue testing purposes a servohydraulic testing machine, INSTRON 8502, were utilized to test the samples under strain-controlled condition with a 
strain-ratio of $\mathrm{R}=-1$ and at $0.1 \mathrm{~Hz}$ frequency (Figure 4). This figure indicates the apparatus used in this study as well as a sample under low cycle fatigue loading while compression and its failure after some cycles.

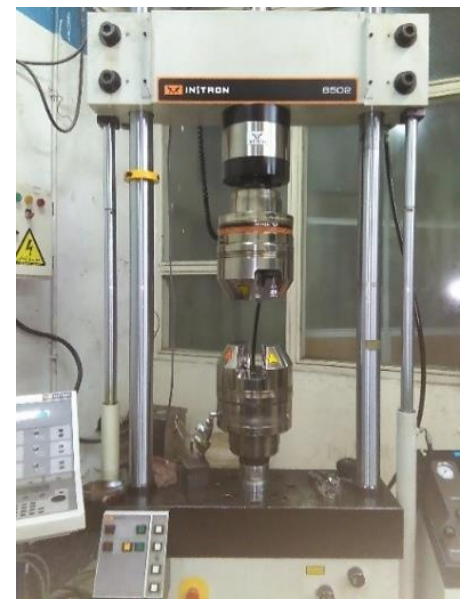

(a)

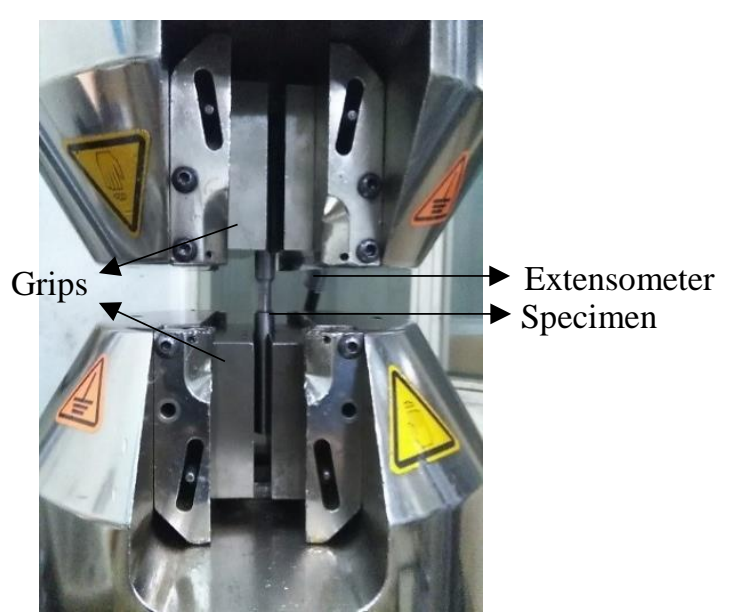

(b)

Figure 4. Low cyclic fatigue test: (a) The fatigue testing machine and (b) one specimen under low cycle fatigue test.

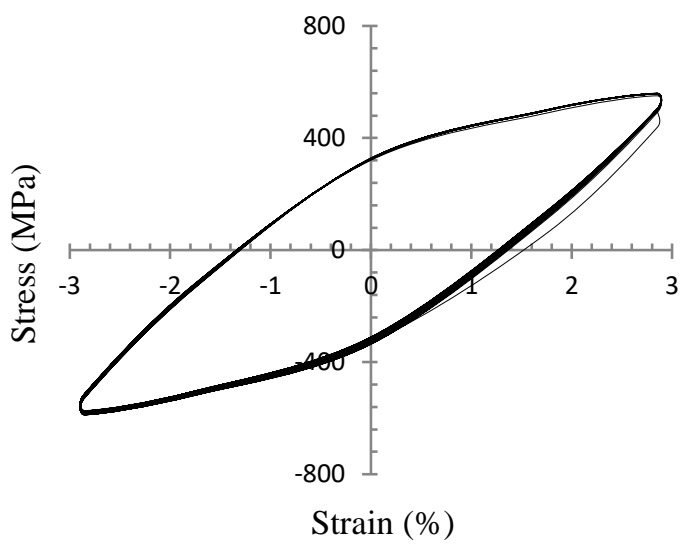

(a)

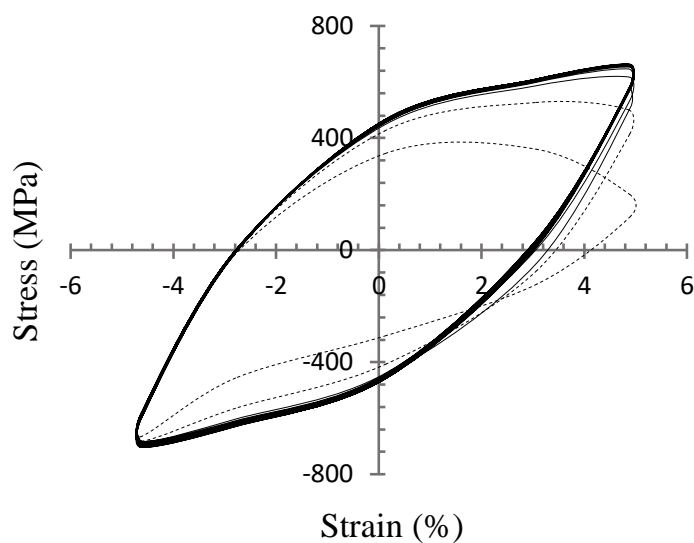

(b)

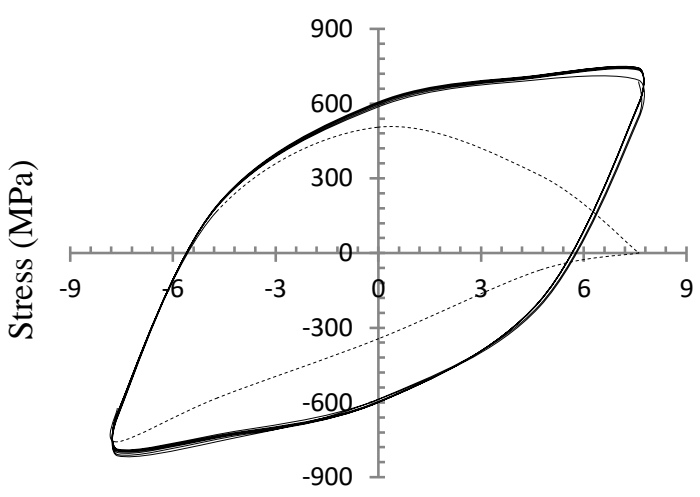

Strain $(\%)$

(c)

Figure 5. Experimentally acquired hysteresis curves of samples under strain-controlled tension/compression loading, enduring a fixed maximum strain of, (a) $3 \%$, (b) $5 \%$ and (c) $8 \%$.

After testing the specimens three curves of stabilized hysteresis loops were obtained for the purpose of calibrating them in order to extract some worthwhile data vital for plotting the curve, to which Eq. (4) should be fitted. Figures 5a-c show the hysteresis curves acquired from strain-controlled low cycle fatigue tests at 3,5 and $8 \%$ strains. It is worth noting that the loops of this figure shown in dash line, indicate the failure of the samples under low cycle fatigue loading. 


\section{RESULTS AND DISCUSSION}

\section{Methodology and Calibration}

To extract the parameters of the Chaboche nonlinear kinematic hardening model, it is needed to calibrate some quantities obtained from stabilized hysteresis stress/strain curves, whereas two other parameters related to the Voce isotropic hardening law are directly found by fitting a relevant equation to the monotonic stress/strain curve. Parameters $\mathrm{A}$ and $\mathrm{b}$, related to the isotropic part of combined hardening model, defined by Eq. (2), can be calculated by fitting the equation to the data obtained from the plastic part of the monotonic stress/strain curve (Figure 6(a)). Moreover, parameters $\mathrm{C}$ and $\gamma$ should be obtained by fitting Eq. (4) to the curve plotted using three data extracted from three stabilized hysteresis loops of material under low cycle fatigue tests [21] (Figure 6(b)).

$$
\frac{\Delta \sigma}{2}-k=\frac{C}{\gamma} \tanh \left(\gamma \frac{\Delta \varepsilon^{p l}}{2}\right)
$$

where $\mathrm{C}$ and $\gamma$ are material constants and $\mathrm{k}$ shows the initial yield stress.

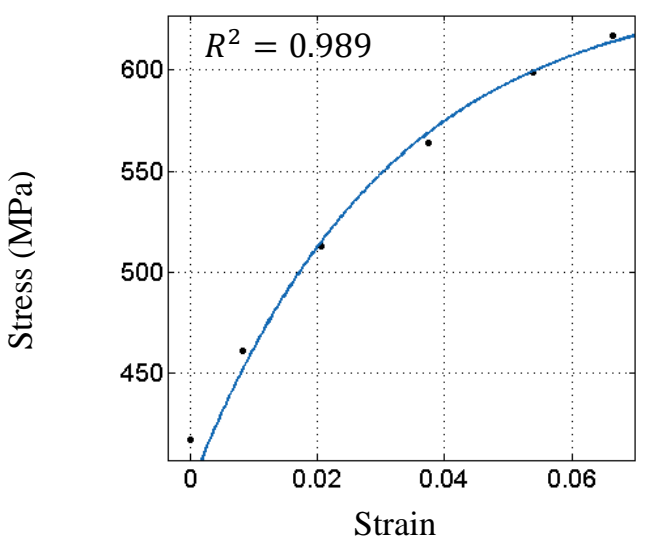

(a)

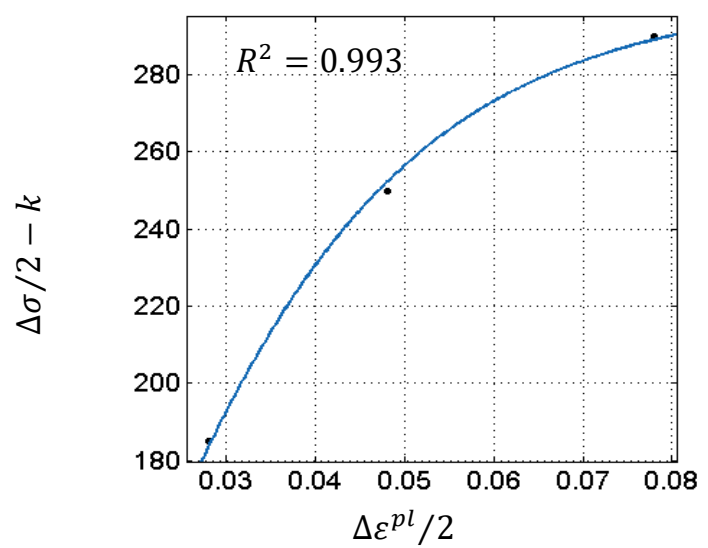

(b)

Figure 6. Curves fitted to the extracted data in order to obtain the constants of nonlinear combined hardening: (a) A and b, (b) C and $\gamma$.

To survey the procedure used to extract three points from three stabilized hysteresis loops, it is helpful to focus on the schematic indicated in Figure 7, for the sake of simplicity. The following procedure mentioned for this figure was repeated for the curves obtained from strain-controlled low cycle fatigue tests of this study, demonstrated in Figure 5. The procedure has some steps as follows [22].

First of all, by subtracting the amount of $\sigma_{s}^{0} / E$ from each datum of each hysteresis curve, a modified curve of hysteresis loops can be plotted, similar to that shown in Figure 7. From this figure, half of the elastic domain, $\mathrm{k}$, and stress range, $\Delta \sigma$, as well as half of the plastic strain range, $\Delta \varepsilon^{p l} / 2$, can be extracted. Then for each hysteresis curve, one pair of data, $\left(\Delta \varepsilon^{p l} / 2, \Delta \sigma / 2-k\right)$, should be calculated and defined as a point of new diagram, for which curve fitting technique should be applied. With three computed pairs of data extracted from three modified hysteresis curves, a new diagram of $\Delta \sigma / 2-k$ versus $\Delta \varepsilon^{p l} / 2$ can be drawn. Eventually, Eq. (4) should be fitted to the aforementioned three points so as to find parameters $\mathrm{C}$ and $\gamma$.

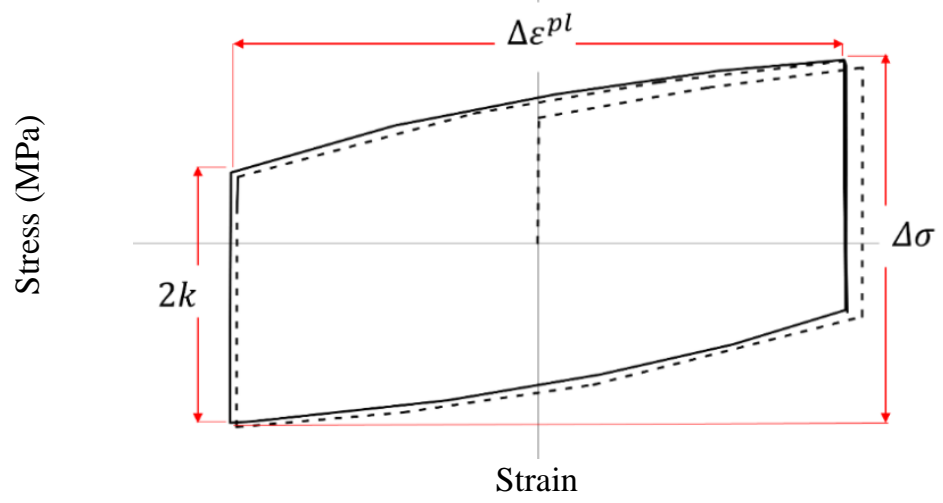

Figure 7. Modified hysteresis loop. 
All parameters extracted from the tests were gathered in Table 2, in brief. These practical parameters can be utilized to model the material in the numerical simulations carried out in commercial finite element packages. This is because the Chaboche nonlinear kinematic hardening not only is one of the most assessed models but also it is almost employed in almost all finite element packages to simulate the kinematic hardening phenomenon in the metallic materials.

Table 2. Obtained parameters of the combined hardening model for steel CK45.

\begin{tabular}{ccc}
\hline Parameter & Definition & Quantity \\
\hline$\sigma_{s}^{0}$ & Initial yield stress, Eq. (2) & $393[\mathrm{MPa}]$ \\
$\mathrm{A}$ & Constant, Eq. (2) & $32.74[\mathrm{MPa}]$ \\
$\mathrm{b}$ & Constant, Eq. (2) & 249.8 \\
$\mathrm{C}$ & Constant, Eq. (3) & $15260[\mathrm{MPa}]$ \\
$\gamma$ & Constant, Eq. (3) & 50.8 \\
\hline
\end{tabular}

Moreover, in order to be more precise in choosing the material, steel CK45, the percentage of the elements composing the material used in manufacturing the samples were extracted through the quantometry test beforehand. Table 3 shows the weight percentage of each element in the material.

Table 3. Chemical composition of CK45 steel (\%).

\begin{tabular}{cccccccccc}
\hline \multicolumn{10}{c}{ Elements } \\
\hline $\mathbf{C}$ & $\mathbf{S i}$ & $\mathbf{M n}$ & $\mathbf{P}$ & $\mathbf{S}$ & $\mathbf{N i}$ & $\mathbf{C r}$ & $\mathbf{M o}$ & $\mathbf{C u}$ & $\mathbf{N}$ \\
0.451 & 0.215 & 0.55 & 0.017 & 0.024 & 0.11 & 0.05 & 0.011 & 0.10 & - \\
\hline
\end{tabular}

\section{Numerical Simulation}

Verification of the method used in this study as well as the accuracy of the acquired parameters of the combined hardening, has been accomplished through two user defined subroutines UMAT written in FORTRAN which were employed into a commercial finite element package ABAQUS. The subroutines of this study were prepared according to two models of hardening, isotropic and combined ones, to perform a comparison between the experimental results and the outcomes of the implemented method. Figure 8 indicates the results of the subroutines at the same conditions as the experimental test under a strain-controlled loading at $8 \%$. The results show a good agreement between the experimental results and those from calculated parameters of the combined hardening model, however, simple isotropic hardening model underestimated the results.

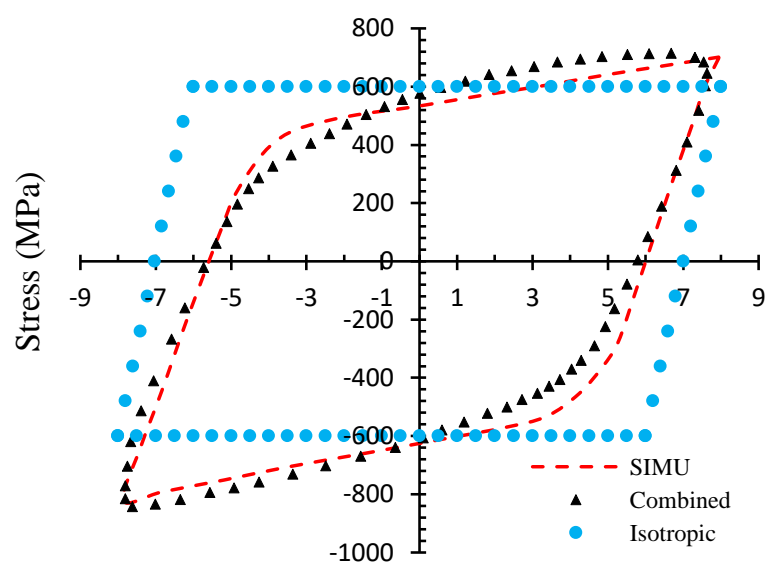

Strain $(\%)$

Figure 8. Hysteresis curves of samples under strain-controlled tension/compression loading, enduring a fixed maximum strain of $8 \%$. It shows a comparison between data obtained from two different user defined subroutines and finite element analysis.

For the guarantee of the exactness of the results obtained in the previous section, a sample identical to that used in experiments was simulated in ABAQUS/Explicit, under strain-controlled cyclic loading and by using the obtained parameters of the nonlinear combined hardening model in order for a precise material definition. All boundary conditions 
are the same as experiments, at which one side of the sample is fully fixed inside the fixture while the other side is experiencing a strain-controlled, uniaxial, tension-compression loading representing low cycle fatigue test. To simulate such kind of loading in ABAQUS, plastic behavior of the material was modeled using the parameters of combined hardening related to the cyclic loading. In order to model the loading in such a way that the strain could be constant, the amount of applied displacement was updated in the software at the beginning of each step of loading, and subsequently the amount of displacement would be increased considering the new length of the sample by which strain-controlled loading condition could be guaranteed. In other words, the length of the sample as an output at the end of each step, was used to calculate the amount of the applied displacement in the following step considering the constant amount of the strain. For this purpose, two farthest points located in both ends of the gage part of the sample were considered as the boarders of the gage, and consequently the distance between them gives the updated length of the gage at the end of each step. This method is practical due to the nature of the loading which is of low cyclic fatigue type and to the fact that the hysteresis loops of stress/strain data reach stabilization state after a few cycles, especially in high amount of strain applied to the samples. Meshing pattern used in the finite element analyses was obtained by trial and error procedure to best simulate the behavior of the sample under tension-compression strain-controlled loading. Mesh sensitivity study was done and a mesh size of $1 \mathrm{~mm}$ was found to be used in parts of high stress concentrations at the end of the gage part. In addition a mesh size of $0.5 \mathrm{~mm}$ used in the gage part to better capture the cyclic behavior of the material. Meshing pattern used in the finite element analyses of the research was shown in Figure 9. The sample utilized some 8-node linear brick, reduced integration, and hourglass control elements, demonstrated in ABAQUS documentation by C3D8R.

Figure 9 demonstrates the sample analyzed numerically, from which the curve denoted by SIMU, shown in the Figure 8 , can be drawn. It shows a stabilized hysteresis curve extracted from the numerical simulation of the sample experiencing strain-controlled loading with a magnitude of $8 \%$. Comparison between Figures 5c and 8, reflects a good agreement between experimental data and numerical investigations. It is worth noting that, considering the placement of a large portion of the sample into the grips of the testing machine (Figure 4) and for the sake of simplicity in the numerical investigations, the parts in the two ends of the sample placed in the fixtures were neglected in the finite element simulation because these parts are fixed into the fixtures and the displacement was applied only in the immediate cross-sectional areas of the sample out of the fixtures, which is the justification for this assumption. Moreover, the stress distribution in the sample as well as the quantities of the endured stresses can be observed in Figure 9.
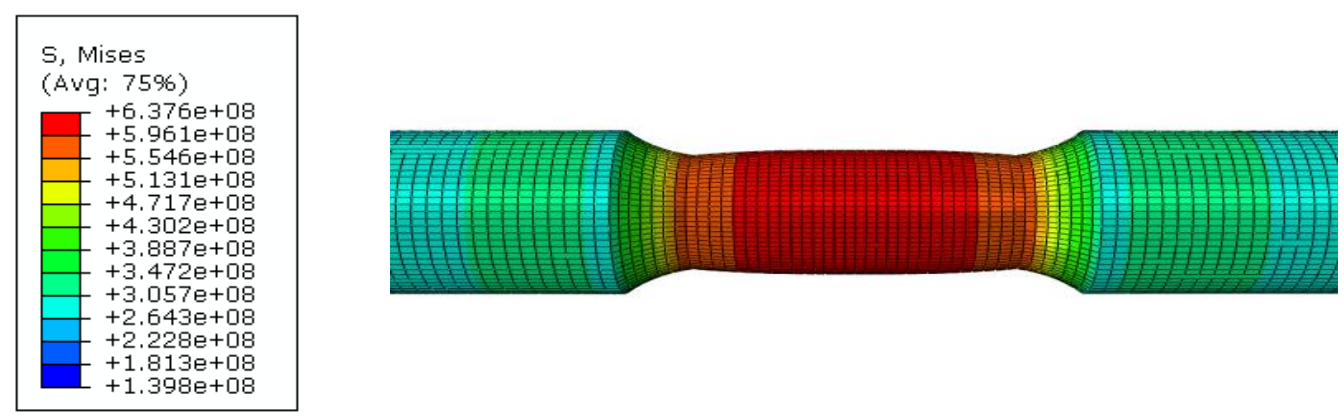

Figure 9. Numerical simulation of the sample under $8 \%$ strain while compression. The middle part of the sample including the gage part was shown in the figure.

\section{CONCLUSION}

The study demonstrated the procedure to obtain the parameters involved in the Chaboche nonlinear kinematic hardening model and Voce isotropic hardening model, along with all experiments necessary for obtaining them. One monotonic stress/strain curve obtained from uniaxial tensile test in addition to three stabilized hysteresis loops extracted from three tension-compression strain-controlled low cyclic fatigue tests, were utilized in order to compute the constants of the combined hardening model. To check the validity of the procedure used in this research as well as the accuracy of the acquired parameters used in the combined hardening model, two user defined subroutines UMAT written in FORTRAN were utilized along with a finite element analysis of a sample of the same dimensions as the one used in the experiments under loading conditions identical to the experiments. The subroutine of combined hardening reflects a stress-strain response really close to experimental data, much better than the subroutine of isotropic hardening does. In the finite element analysis the outcomes showed a good agreement between the numerical and experimental works, as a justification for the accuracy of the procedure used to calculate the constants of the combined model.

\section{REFERENCES}

[1] A. S. Khan and S. Huang, Continuum Theory of Plasticity. Wiley, 1995.

[2] F. A. McClintock and A. S. Argon, Mechanical behavior of materials. Addison-Wesley Pub. Co., 1966. 
[3] K. P. Li, W. P. Carden, and R. H. Wagoner, "Simulation of springback," International Journal of Mechanical Sciences, vol. 44, no. 1, pp. 103-122, 2002/01/01/ 2002, doi: https://doi.org/10.1016/S0020-7403(01)00083-2.

[4] W. Ramberg, W.R. Osgood, and S. United, "A. National Advisory Committee for, Description of stress-strain curves by three parameters," National Advisory Committee for Aeronautics, 1943.

[5] H. Ziegler, "A Modification of Prager's Hardening Rule," Quarterly of Applied Mathematics, vol. 17, no. 1, pp. 55-65, 1959. [Online]. Available: www.jstor.org/stable/43634629.

[6] W. Prager, "The Theory of Plasticity: A Survey of Recent Achievements," Proceedings of the Institution of Mechanical Engineers, vol. 169, no. 1, pp. 41-57, 1955/06/01 1955, doi: 10.1243/PIME_PROC_1955_169_015_02.

[7] C. O. Frederick and P. J. Armstrong, "A mathematical representation of the multiaxial Bauschinger effect," Materials at High Temperatures, vol. 24, no. 1, pp. 1-26, 2007/01/01 2007, doi: 10.1179/096034007X207589.

[8] E. Voce, "A practical strain hardening function," Metallurgia, vol. 51, pp. 219-226, 1955.

[9] X. Zhuang, Y. Ma, and Z. Zhao, "Fracture prediction under nonproportional loadings by considering combined hardening and fatigue-rule-based damage accumulation," International Journal of Mechanical Sciences, vol. 150, pp. 51-65, 2019/01/01/ 2019, doi: https://doi.org/10.1016/j.ijmecsci.2018.09.057.

[10] L. Zhao, L. Xu, Y. Han, H. Jing, and Z. Gao, "Modelling creep-fatigue behaviours using a modified combined kinematic and isotropic hardening model considering the damage accumulation," International Journal of Mechanical Sciences, vol. 161162, p. 105016, 2019/10/01/2019, doi: https://doi.org/10.1016/j.ijmecsci.2019.105016.

[11] M. Aghaei and S. Ziaei-Rad, "A micro mechanical study on DP600 steel under tensile loading using Lemaitre damage model coupled with combined hardening," Materials Science and Engineering: A, vol. 772, p. 138774, 2020/01/20/ 2020, doi: https://doi.org/10.1016/j.msea.2019.138774.

[12] F. Yoshida, H. Hamasaki, and T. Uemori, "Modeling of anisotropic hardening of sheet metals including description of the Bauschinger effect," International Journal of Plasticity, vol. 75, pp. 170-188, 2015/12/01/ 2015, doi: https://doi.org/10.1016/j.ijplas.2015.02.004.

[13] E.-H. Lee, H. Choi, T. B. Stoughton, and J. W. Yoon, "Combined anisotropic and distortion hardening to describe directional response with Bauschinger effect," International Journal of Plasticity, vol. 122, pp. 73-88, 2019/11/01/ 2019, doi: https://doi.org/10.1016/j.ijplas.2019.07.007.

[14] E.-H. Lee, T. Stoughton, and J. Yoon, "A yield criterion through coupling of quadratic and non-quadratic functions for anisotropic hardening with non-associated flow rule," International Journal of Plasticity, vol. 99, 09/01 2017, doi: 10.1016/j.ijplas.2017.08.007.

[15] E.-H. Lee, T. B. Stoughton, and J. W. Yoon, "A new strategy to describe nonlinear elastic and asymmetric plastic behaviors with one yield surface," International Journal of Plasticity, vol. 98, pp. 217-238, 2017/11/01/ 2017, doi: https://doi.org/10.1016/j.ijplas.2017.08.003.

[16] J. L. Chaboche, "Time-independent constitutive theories for cyclic plasticity," International Journal of Plasticity, vol. 2, no. 2, pp. 149-188, 1986/01/01/ 1986, doi: https://doi.org/10.1016/0749-6419(86)90010-0.

[17] J. L. Chaboche, "Constitutive equations for cyclic plasticity and cyclic viscoplasticity," International Journal of Plasticity, vol. 5, no. 3, pp. 247-302, 1989/01/01/ 1989, doi: https://doi.org/10.1016/0749-6419(89)90015-6.

[18] J. L. Chaboche and G. Rousselier, "On the Plastic and Viscoplastic Constitutive Equations-Part I: Rules Developed With Internal Variable Concept," Journal of Pressure Vessel Technology, vol. 105, no. 2, pp. 153-158, 1983, doi: 10.1115/1.3264257.

[19] M. Zehsaz, F. V. Tahami, and H. Akhani, "Experimental determination of material parameters using stabilized cycle tests to predict thermal ratchetting," vol. 78, pp. 17-30, 01/01 2016.

[20] I. Sheldon, "Chaboche Nonlinear Kinematic Hardening Model," STI0805, ANSYS Release: 11, 2008.

[21] "Advanced Metal Plasticity," Lecture 2-@ 2011 ANSYS, Inc., 2012.

[22] G. B. Broggiato, F. Campana, and L. Cortese, "The Chaboche nonlinear kinematic hardening model: calibration methodology and validation," Meccanica, vol. 43, no. 2, pp. 115-124, 2008/04/01 2008, doi: 10.1007/s11012-008-9115-9. 